\title{
Features Analysis of Partial Demagnetization Faults in the 6-phase PM Synchronous Generator with Rectifier Load System
}

\author{
Binghui Wang ${ }^{1,}$, Yuguang Sun ${ }^{1, b}$ \\ ${ }^{1}$ Department of Electrical Engineering, Tsinghua University, Beijing 100084, China; \\ ahbwzztx@163.com, bsunyuguang98@mails.tsinghua.edu.cn
}

\begin{abstract}
Keywords: 6-phase permanent magnet synchronous genrator, partial demagnetization faults, FEM combined with electric circuit method.
\end{abstract}

\begin{abstract}
This paper focuses on partial demagnetization faults (PDMF) in the 6-phase permanent magnet synchronous generator (PMSG) with rectifier load system. In the study, a multi-loop combined with finite element method (FEM) mathematical model is presented. The multi-loop model utilizes transformation matrix to describe the dynamics of actual circuit topology, which is influenced by on-and-off states of diodes. The 2-D FEM is employed to calculate magnetic field excited by the PM rotor and induced electromotive force (EMF) in stator coils. Simulation and experimental results imply that phase voltage, phase current, dc load voltage and dc load current decline when PDMF occurs. What's more, significant unbalanced current will appear between parallel connected windings if the machine has two parallel branches in a phase winding. The analysis of the paper provides meaningful reference to on-line monitoring and protection for demagnetization faults in the 6-phase PMSG.
\end{abstract}

\section{Introduction}

6-phase permanent magnet synchronous generator (PMSG) have attracted appreciable interest in various applications, such as wind generation, electric vehicles, due to its high quality and high power density. Besides, the 6-phase PMSG with two parallel branches in a phase winding can be applied to generate electromagnetic pulse. Similar to general PM machines, performance and efficiency of the 6-phase PMSG influenced by PM rotor greatly. Demagnetization faults can lead to increasing vibration and more copper losses ${ }^{[1]}$. Therefore, it is of important reality and economy significance to analysis characteristics of demagnetized 6-phase PMSG.

Several papers about the theme have been reported. FEM combined with electric circuit method has been utilized to establish circuit equations of 6-phase PMSG in d-q coordinate system [2]. Not only can the method calculate magnetic field accurately, but it takes harmonic field and iron saturation into consideration. Transformation matrix has been used to depict the rectifier bridge working states of 12-phase synchronous generator when internal stator winding faults in the machine was analyzed [3], the matrix turned out to be flexible and suitable for conditions that multiple rectifier bridges working in series or parallel at the same time. Demagnetization faults features are extracted by means of comparing stator current [4], stator voltage [5] and magnetic field [6] before and after the fault, and finite element method (FEM) has been proved to be an effective tool to simulate the electrical quantities according to these researches.

This paper focuses on partial demagnetization faults (PDMF) in 6-phase PMSG. A mathematical model considering demagnetization faults will be presented making use of the FEM combined with electric circuit method. In the model, dynamics of actual circuit topology will be described by transformation matrix with on-and-off states of diodes in view, magnetic field and related electrical quantities will be obtained by taking advantage of FEM.

\section{Mathematical model for partial demagnetized faults in 6-phase PMSG}

In a general way, this paper takes 6-phase PMSG having two parallel branches in per phase as concrete research object, the two parallel branches away from each other a pair of poles in space. 
Principles of the system is shown in Fig. 1. It can be find that the stator windings are equivalent to two groups of $\mathrm{Y}$ type three-phase symmetric windings apart $30^{\circ}$ electrical degree from each other. Each $\mathrm{Y}$ type three-phase symmetric windings connects with an uncontrolled rectifier bridge and the two $\mathrm{Y}$ type three-phase windings power dc load in parallel connection.

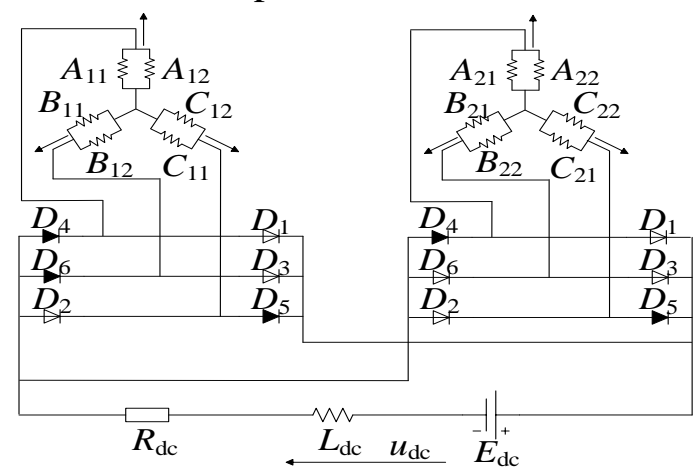

Fig. 1 Principles for 6-phase PMSG having two parallel branches in per phase with rectifier load system

Multi-loop model of the 6-phase PMSG with rectifier load system

\section{1) Voltage equation of stator branches}

In this paper, reference direction for voltage, current and flux linkages is specified as below: voltage is positive when it points from neutral to terminal; current is positive when it flows from neutral to terminal; positive current excites positive flux linkage. Viewing stator branch windings as basic research units, voltage equation of stator branches can be expressed as follows:

$$
\boldsymbol{U}_{\mathrm{s}}=p \psi_{\mathrm{s}}+p \boldsymbol{\psi}_{\mathrm{pm}}+\boldsymbol{I}_{\mathrm{s}} \boldsymbol{R}_{\mathrm{s}}
$$

where $p$ is derivative operator $\mathrm{d} / \mathrm{d} t ; \quad \boldsymbol{U}_{\mathrm{s}}=\left[u_{A_{11}}, u_{A_{12}}, \ldots, u_{C_{22}}\right]^{T}, \quad \boldsymbol{I}_{\mathrm{s}}=\left[i_{A_{11}}, i_{A_{12}}, \ldots, i_{C_{22}}\right]^{T} \quad$ and $\boldsymbol{R}_{\mathrm{s}}=\operatorname{diag}\left[r_{A_{11}}, r_{A_{12}}, \ldots, r_{C_{22}}\right]$, represent stator branch voltage vector, stator branch current vector and stator branch resistance matrix respectively; $\boldsymbol{\psi}_{\mathrm{pm}}$ is PM flux linkage vector, which can be obtained by FEM; $\psi_{\mathrm{s}}$ is armature coil-induced flux linkage vector and it can be calculated by:

$$
\psi_{\mathrm{s}}=\boldsymbol{M}_{\mathrm{s}} \boldsymbol{I}_{\mathrm{s}}
$$

where $\boldsymbol{M}_{\mathrm{s}}$ is stator branch inductance matrix and $\boldsymbol{M}_{\mathrm{s}}=\left[\begin{array}{ccccc}L_{A_{11}} & M_{A_{11}, A_{12}} & \cdots & M_{A_{11}, C_{12}} & M_{A_{11}, C_{22}} \\ M_{A_{12}, A_{11}} & L_{A_{12}} & \cdots & M_{A_{12}, C_{12}} & M_{A_{12}, C_{22}} \\ \vdots & \vdots & \ddots & \vdots & \vdots \\ M_{C_{12}, A_{11}} & M_{C_{12}, A_{12}} & \cdots & L_{C_{12}} & M_{C_{12}, C_{22}} \\ M_{C_{22}, A_{11}} & M_{C_{22}, A_{12}} & \cdots & M_{C_{22}, C_{12}} & L_{C_{22}}\end{array}\right]$.

This paper calculates $\boldsymbol{M}_{\mathrm{s}}$ by adopting permeability frozen method to take iron saturation due to PM into account, which learns from [7].

By substituting (2) in (1), the voltage equation of stator branches results in the following:

$$
\boldsymbol{U}_{\mathrm{s}}=\left(p \boldsymbol{M}_{\mathrm{s}}\right) \boldsymbol{I}_{\mathrm{s}}+\boldsymbol{M}_{\mathrm{s}}\left(p \boldsymbol{I}_{\mathrm{s}}\right)+\boldsymbol{I}_{\mathrm{s}} \boldsymbol{R}_{\mathrm{s}}+\boldsymbol{E}_{\mathrm{pm}}
$$

Where $\boldsymbol{E}_{\mathrm{pm}}$ is branch induced EMF vector. Calculation of $\boldsymbol{E}_{\mathrm{pm}}$ will be illustrated in 1.2 part of this paper.

\section{2) Voltage equation of dc load}

Voltage equation of dc load can be expressed as follows:

$$
u_{\mathrm{dc}}=i_{\mathrm{dc}} R_{\mathrm{dc}}+L_{\mathrm{dc}} \frac{\mathrm{d} i_{\mathrm{dc}}}{\mathrm{d} t}+E_{\mathrm{dc}}
$$

where $R_{\mathrm{dc}}, L_{\mathrm{dc}}$ and $E_{\mathrm{dc}}$ are resistive load, inductive load and back-emf load (e.g. a battery), respectively.

\section{3) Multi-loop model of the system}

Combining (3) with (4), branch voltage equation of the system is given by: 


$$
\boldsymbol{U}=(p \boldsymbol{M}+\boldsymbol{R}) \boldsymbol{I}+\boldsymbol{M}(p \boldsymbol{I})+\boldsymbol{E}
$$

where $\boldsymbol{U}=\left[\begin{array}{ll}\boldsymbol{U}_{\mathrm{s}}{ }^{T} & u_{\mathrm{dc}}\end{array}\right]^{T}, \boldsymbol{I}=\left[\begin{array}{ll}\boldsymbol{I}_{\mathrm{s}}{ }^{T} & i_{\mathrm{dc}}\end{array}\right]^{T}, \boldsymbol{E}=\left[\begin{array}{ll}\boldsymbol{E}_{\mathrm{pm}}{ }^{T} & E_{\mathrm{dc}}\end{array}\right]^{T}, \boldsymbol{M}=\operatorname{diag}\left[\begin{array}{ll}\boldsymbol{M}_{\mathrm{s}} & L_{\mathrm{dc}}\end{array}\right], \boldsymbol{R}=\operatorname{diag}\left[\begin{array}{ll}\boldsymbol{R}_{\mathrm{s}} & R_{\mathrm{dc}}\end{array}\right]$

To solve the branch voltage equation of the system, actual circuit topology should be considered since branch voltage and branch current are both unknown. As is shown in Fig.1, two parallel branches in per phase constitutes conduction loop physically, what's more, phase windings, rectifier bridge and dc load make up dynamic loops. It's obvious that the former loops have no relationship with rectifier bridge, while the latter loops vary from the on-and-off states of diodes. Counting in on-and-off states of diodes, transformation matrix is applied to depict the dynamics of actual circuit topology in this paper. Rows and columns of the matrix represent circuit loops and electric elements separately, and the elements the matrix are set as 0,1 or -1 depending on whether electric element is relevant with circuit loop and their direction are consistent with each other or not. Taking the circuit topology shown in Fig.1 as example(the on-state diodes and off-state diodes are identified with solid and hollow symbol respectively), the transformation matrix can be presented as follows:

stator branches $\overbrace{}^{\text {dc load }}$

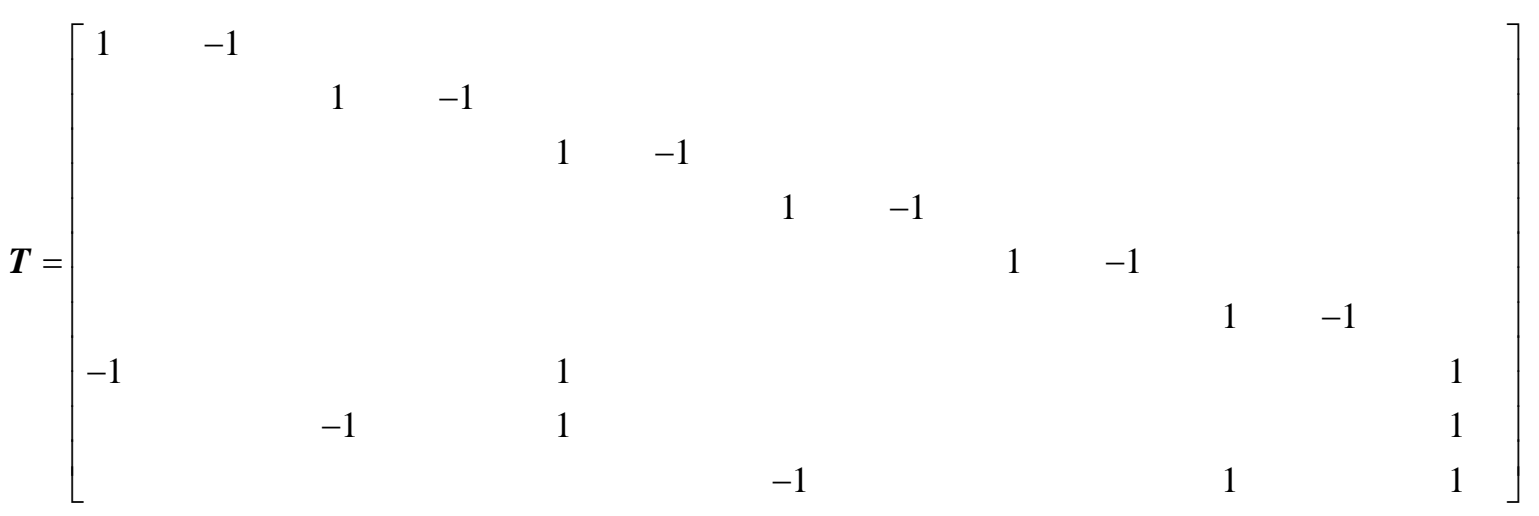

Based on the connecting way of the 6-phase PMSG with rectifier load system showed in Fig. 1, it's can be known that column number of transformation matrix remains constant (the column number is 13 , for the reason that the system contains twelve stator branches and a dc load branch ), and so do the elements of the first six rows. Nevertheless, the row number and elements of the after six rows vary from diodes' on-and-off states of the two rectifier bridges.

By means of the transformation matrix, connections can be constructed between branch vector and loop vector of the system:

$$
\begin{aligned}
& \boldsymbol{U}^{\prime}=\boldsymbol{T} \boldsymbol{U} \\
& \boldsymbol{I}=\boldsymbol{T}^{T} \boldsymbol{I}^{\prime}
\end{aligned}
$$

where $\boldsymbol{U}^{\prime}$ is loop voltage vector and $\boldsymbol{I}^{\prime}$ is loop current vector.

Substituting (6) and (7)into (5) results in multi-loop model of the system:

$$
\boldsymbol{U}^{\prime}=\left(p \boldsymbol{M}^{\prime}+\boldsymbol{R}^{\prime}\right) \boldsymbol{I}^{\prime}+\boldsymbol{M}^{\prime}\left(p \boldsymbol{I}^{\prime}\right)+\boldsymbol{E}^{\prime}
$$

where $\boldsymbol{M}^{\prime}=\mathbf{T} \boldsymbol{M} \boldsymbol{T}^{T}, \boldsymbol{R}^{\prime}=\boldsymbol{T} \boldsymbol{R} \boldsymbol{T}^{T}, \boldsymbol{E}^{\prime}=\boldsymbol{T E}$.

From (8) we can learn that electrical quantities of the system could be simulated if induced EMF have been calculated.

\section{Calculation of induced EMF due to PM by FEM}

Air-gap flux density obtained by FEM can be decomposed into the fundamental and a series of harmonic space components in the rotor coordinate system, which is expressed as follows:

$$
B(x)=\sum_{k} B_{k} \cos \left(k x+\varphi_{k}\right)
$$

where $\boldsymbol{B}_{k}$ and $\varphi_{k}$ are amplitude and phase angle of $k$ harmonic flux density.

Considering magnetic field due to PM has different space cycle on different working conditions, consequently, $k$ has different values when PM is healthy or partial demagnetized. In normal operations, the space cycle of magnetic field is a pair of poles, air-gap flux density only contains 
fundamental and odd harmonics, that is to say, $k$ is equal to $1,3,5$ and so on. While in PDMF conditions, the space cycle of magnetic field is an entire circumference of the machine, air-gap flux density contains integer and fractional harmonics, which means $k$ is equal to $1 / P, 2 / P$, $3 / P$ and so on(where $P$ is the number of pole pairs).

Basing on air-gap flux density, induced EMF in stator coils can be calculated according to the following (taking No.i coil as example):

$$
\begin{aligned}
e_{i}(t)= & \sum_{k} e_{i, k}(t) \\
& =w_{i} \tau \frac{l}{\pi} \omega \sum_{k} B_{k} \sin \left(\frac{k \beta_{\mathrm{s}} \pi}{2}\right) \sin \left(k\left(\omega t-\alpha_{i 0}\right)+\varphi_{k}\right)
\end{aligned}
$$

where $\tau$ is pole pitch; $w_{i}, l, \beta_{\mathrm{s}}$ are turns, axial length, the ratio of coil pitch and pole pitch, for No.i coil, respectively.

Further, induced EMF in stator branches can be gained based on the actual connections of stator windings. Substituting $\boldsymbol{E}_{\mathrm{pm}}$ under demagnetized fault conditions into (8), a multi-loop combined with FEM mathematical model is set up for demagnetization faults in the 6-phase PMSG with rectifier load system.

\section{Simulations and experiments}

\section{Major parameters of a 6-phase PMSG experimental prototype}

A 6-phase PMSG prototype was designed to validate the model presented in section 1 of this paper. For the prototype, pair poles, rated speed and the rated power is 2,1500rpm and $4 \mathrm{KW}$, respectively. Material for prototype PMs is Nd-Fe-B and its coercivity is $812 \mathrm{kA} / \mathrm{m}$. To carry out PDMF experiments conveniently, PMs are buried in the rotor. Geometric model of the prototype is shown in Fig. 2.

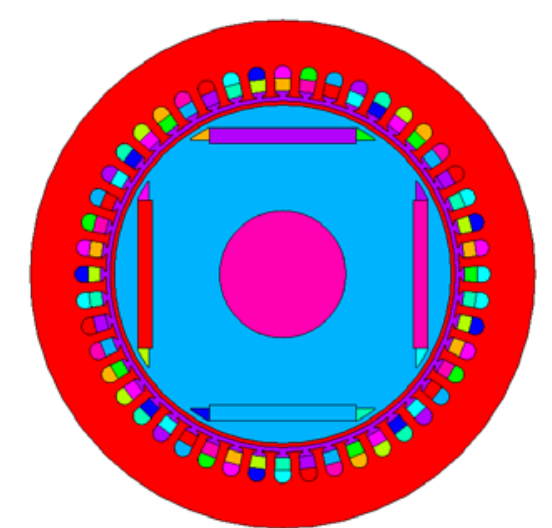

Fig. 2 Geometric model of the 6-phase PMSG experimental prototype.

\section{Simulation and experiment in normal operation}

On the base of the multi-loop combined with FEM mathematical model presented in section 1, performances of the system under normal and PDMF conditions are figured out by simulation algorithm. And corresponding experiments are carried out to validate the proposal. Simulation and experimental results of various electric quantities for healthy machine are shown in Fig. 3 (the speed is $1500 \mathrm{rpm}$ and the dc load is pure resistive load with resistance value is $1.46 \Omega$ ).

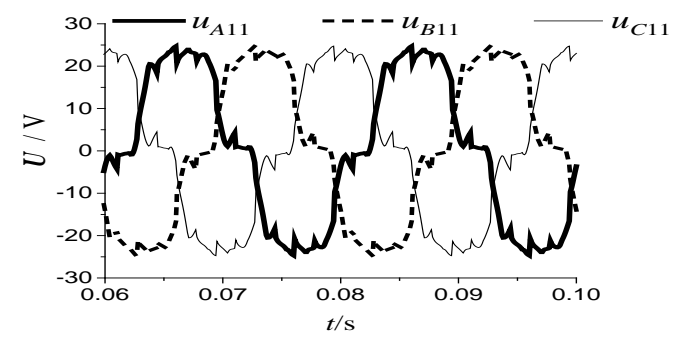

(a)

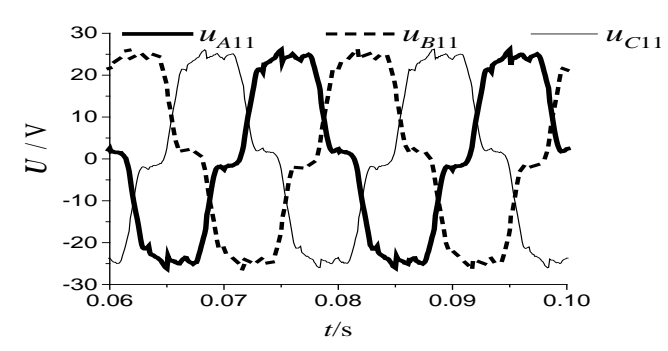

(b) 


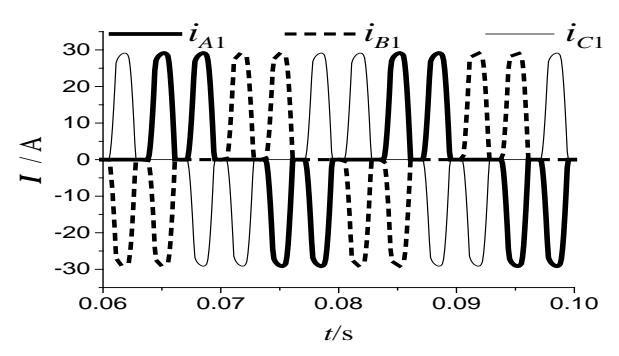

(c)

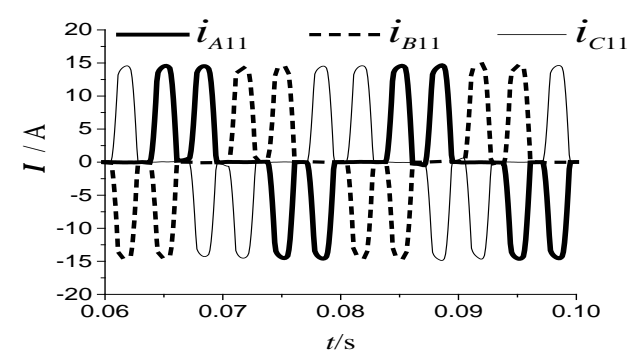

(e)

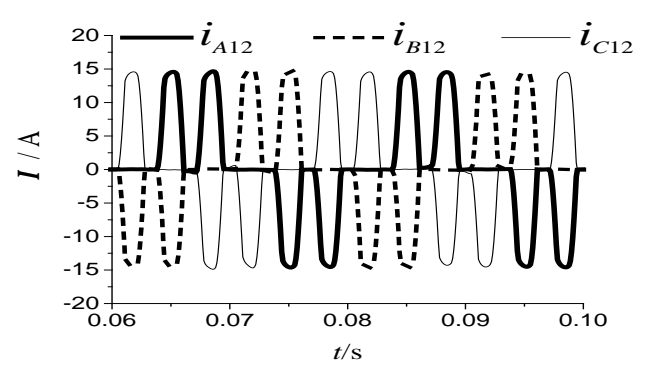

(g)

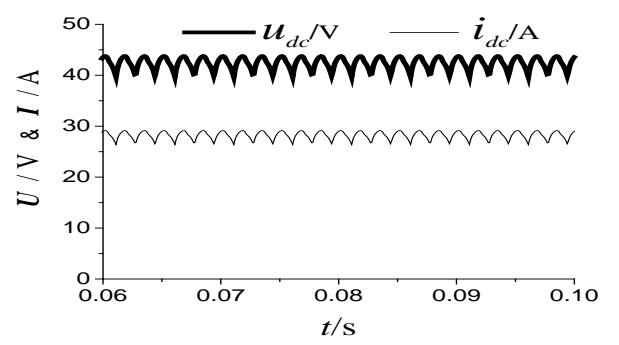

(i)

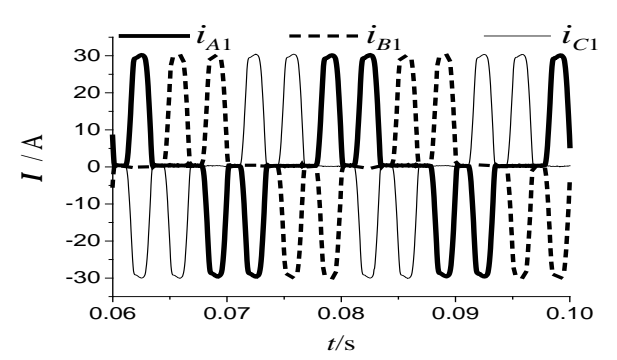

(d)

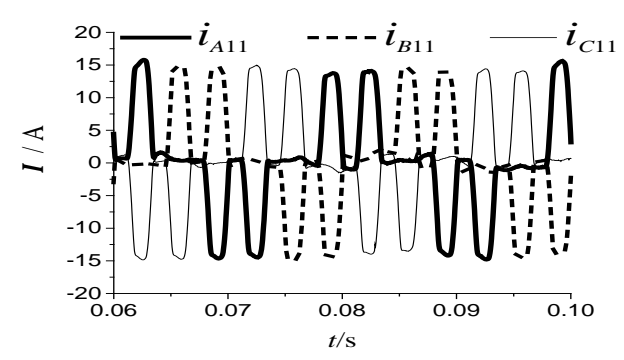

(f)

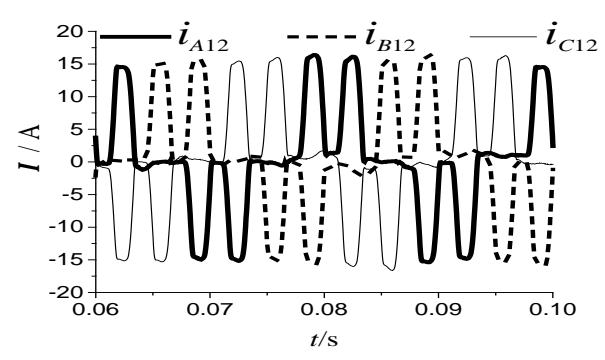

(h)

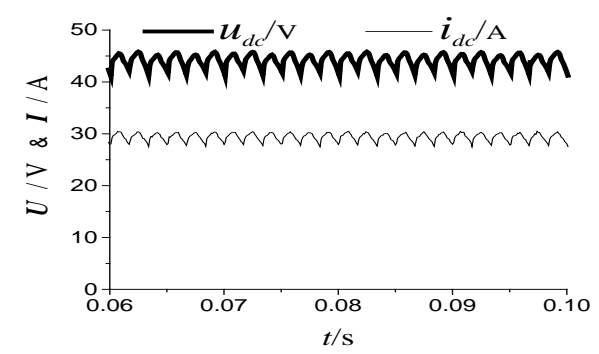

(j)

Fig. 3 Simulation and experimental waveforms of various electric quantities for healthy machine. (a)Simulation voltage waveforms of phase $A_{1}, B_{1}$ and $C_{1}$. (b)Experimental voltage waveforms of phase $A_{1}, B_{1}$ and $C_{1}$. (c)Simulation current waveforms of phase $A_{1}, B_{1}$ and $C_{1}$. (d)Experimental current waveforms of phase $A_{1}, B_{1}$ and $C_{1}$. (e)Simulation current waveforms of branch $A_{11}, B_{11}$ and

$C_{11}$. (f) Experimental current waveforms of branch $A_{11}, B_{11}$ and $C_{11}$. (g)Simulation current waveforms of branch $A_{12}, B_{12}$ and $C_{12}$. (h) Experimental current waveforms of branch $A_{12}, B_{12}$ and $C_{12}$. (i) Simulation waveforms of dc load voltage and dc load current. (j)Experimental waveforms of dc load voltage and dc load current.

As we can see in Fig. 3, three-phase voltages are symmetrical and so are three-phase currents; simulation results of two parallel branch currents in one phase are same and the experimental results are also alike approximately; dc load voltage and current perform 12 times frequency pulse in an electrical cycle. And the simulation and experimental results coincide with each other well.

\section{Simulation and experiment in PDMF operation with a $20 \%$ demagnetized pole}

Simulation and experimental results of various electric quantities for faulty machine with a $20 \%$ demagnetized pole are shown in Fig. 4 (the speed is 1500rpm and the rectifier load is pure resistive load with resistance value is $1.46 \Omega$ ). 


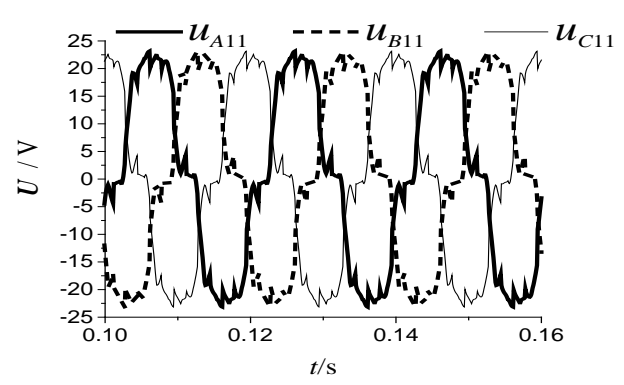

(a)

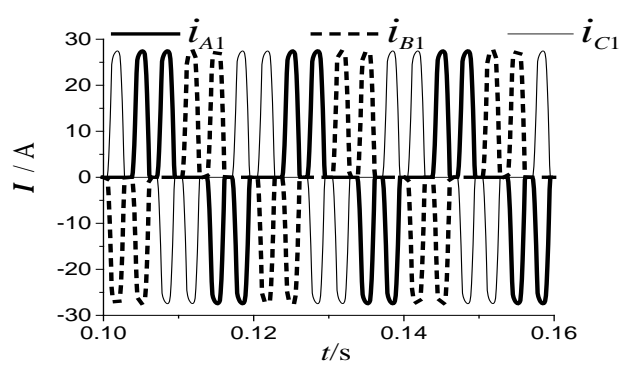

(c)

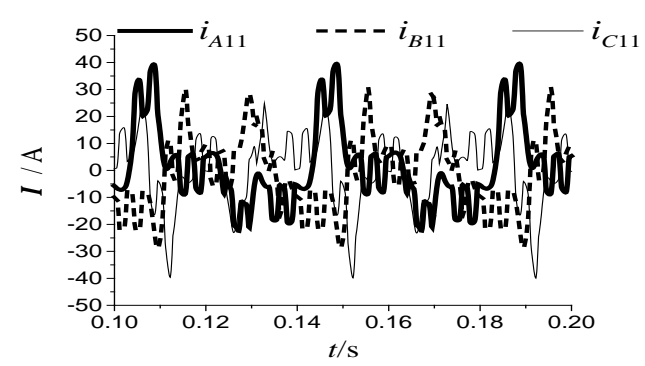

(e)

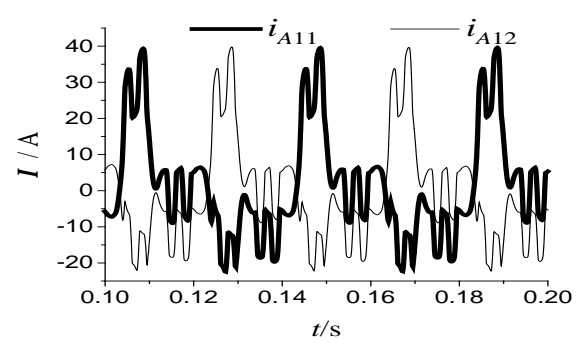

(g)

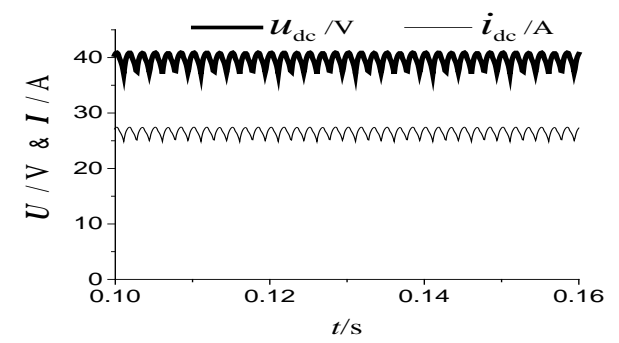

(i)

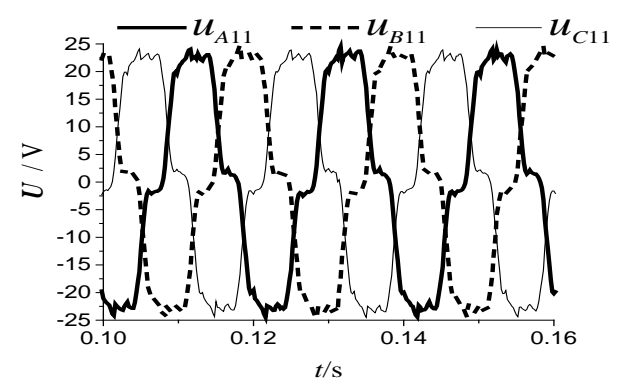

(b)

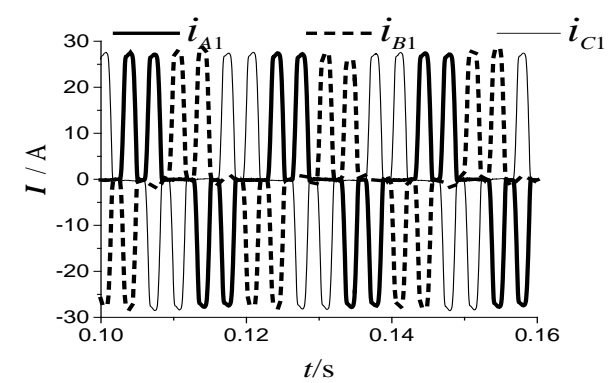

(d)

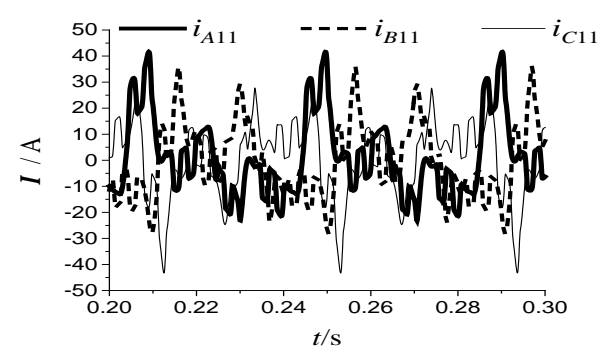

(f)

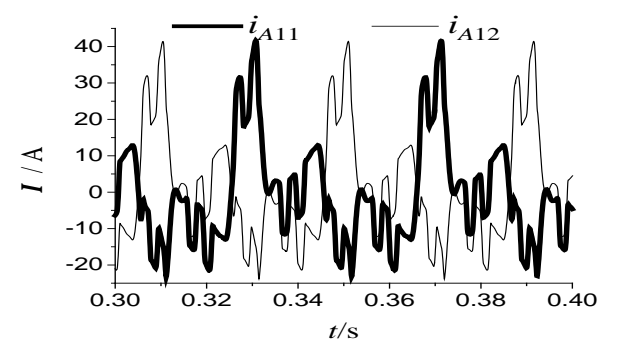

(h)

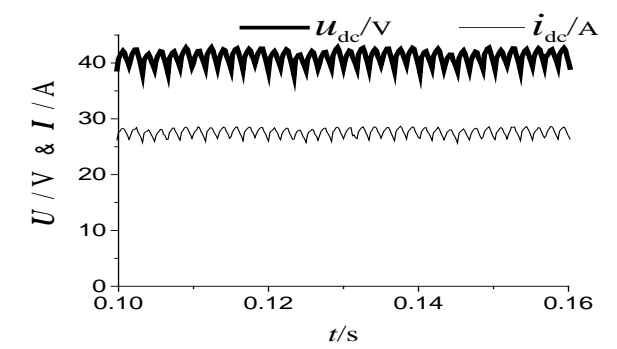

(j)

Fig. 4 Simulation and experimental waveforms of various electric quantities for faulty machine with a 20\% demagnetized pole. (a)Simulation voltage waveforms of phase $A_{1}, B_{1}$ and $C_{1}$. (b)Experimental voltage waveforms of phase $A_{1}, B_{1}$ and $C_{1}$. (c)Simulation current waveforms of phase $A_{1}, B_{1}$ and $C_{1}$.

(d)Experimental current waveforms of phase $A_{1}, B_{1}$ and $C_{1}$. (e)Simulation current waveforms of

branch $A_{11}, B_{11}$ and $C_{11}$. (f)Experimental current waveforms of branch $A_{11}, B_{11}$ and $C_{11}$. (g)

Simulation waveforms of the two parallel branch currents in phase $A_{1}$.(h) Experimental waveforms of the two parallel branch currents in phase $A_{1}$. (i)Simulation waveforms of dc load voltage and dc load current.(j) Experimental waveforms of dc load voltage and dc load current. 
Fig. 4 shows that the simulation results are in better agreement with experimental ones in PDMF operation. When PDMF occurs, it's obvious to find that waveforms of phase voltage, phase current, dc load voltage and dc load current remain similar to healthy ones, while amplitues of these electric quantities decline. Noticely, the most striking fault feature is that waveforms of branch currents distort dramaticly and two branch currents in one phase are not equal to each other at all, which means significant unbalanced current appeared between the parallel stator branch windings.

\section{Summary}

In this paper, a multi-loop combined with FEM mathematical model is presented to analysis PDMF features in 6-phase PMSG with rectifier load system. Simulation results of various electric quantities coincide with experimental ones well, which verifies the accuracy and effectiveness of the model. In PDMF conditions, phase voltage, phase current, dc load voltage and dc load current decrease remarkablely, resulting in dc load ability of the machine reduction. Moreover, PDMF will lead to notable unbalanced current inside the phase windings if the machine has two parallel branches in a phase, which causes more copper losses and threathes security and stability of the machine. Research in the paper supplies vaulable theory reference to on-line monitoring and protection for demagnetization faults in the 6-phase PMSG with rectifier load system.

\section{References}

[1] Xiaomin Lu, K. Lakshmi Varaha Iyer, Kaushik Mukherjee, et al. Study and Detection of Demagnetization in Line Start Permanent Magnet Synchronous Machines Using Artificial Neural Network. International Conference on Electrical Machines and Systems, 2012,1-6.

[2] Jian Liu. Reserch on Control Technologies of Six-phase Permenent Magenet Synchronous Genetator.2014.

[3] Yuguang Sun, Ziguo Huang, Li Chen, et al. Calculation of Stator Winding Inter-turn short Circuit in 12-phase synchronous Generators with Rectifier Load System. TRANSACTIONS OF CHINA ELECTROTECHNICAL SOCIETY, 2014, 29(3):27-32.

[4] Leran Zhao. Research on the different demagnetized of off-grid permanent magnet wind generator operating performance. 2013.

[5] Julio-CésarUrresty, Jordi-Roger Riba, Miguel Delgado, et al. Detection of Demagnetization Faults in Surface-Mounted Permanent Magnet Synchronous Motors by Means of the Zero-Sequence Voltage Component. IEEE TRANSACTIONS ON ENERGY CONVERSION, 2012, 27(1):42-50

[6] Jordi-Roger Riba Ruiz, Javier A. Rosero, Antonio Garcia Espinosa, et al. Detection of Demagnetization Faults in permanent-Magnet Synchronous Motors Under Nonstationary Conditions. IEEE TRANSACTIONS ON MAGNETICS, 2009, 45(7):2961-2968.

[7] Jin Hur. Characteristic Analysis of Interior Permanent-Magnet Synchronous Motor in Electrohydraulic Power Steering Systems. IEEE TRANSACTIONS ON INDUSTRIAL ELECTRONICS, 2008, 55 (6):2316-2322. 